\title{
Comparative Studying for Opinion Mining and Sentiment Analysis Algorithms and Applications
}

Rana Z. Alobaidy

College of Computer Sciences and mathematics

Ghaydaa A.A. Al-Talib

ghaydabdulaziz@uomosul.edu.iq

University of Mosul, Mosul, Iraq

\section{Received on: 18/03/2018}

Accepted on: 27/08/2018

\section{ABSTRACT}

The amount of the available data increases the ability to analyze and understand. The internet revolution has added billions of customer's review data in its depots. This has given an interest in sentiment analysis and opinion mining in the recent years. People have to depend on machines to classify and process the data as there are terabytes of review data in stock of a single product. So that prediction customer sentiments is very important to analyze the reviews as it not only helps in increasing profits but also goes a long way in improving and bringing out better products. In this paper, we present a survey regarding the presently available techniques and applications that appear in the field of opinion mining, such as , economy, security, marketing, spam detection, decision making, and elections expectation.

Keywords: Data mining, social media, SVM.

$$
\text { رنا زهير عبد الغني العبيدي مقارنة لنيات التنقيب في الآراء وتحليل العواطف وتطبيقاتها }
$$

ghaydabdulaziz@uomosul.edu.iq

$$
\text { جلية علوم الحاسوب والرياضيات الدول، الدوصل، العراق }
$$

تاريخ قبول البحث: 2018108127

تاريخ استلام البحث: 2018103118 - (203

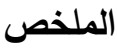

إن كمية البيانات المتوافرة زادت من القابلية على التحليل والفهم , وأضافت ثورة الانترنت البلايين من وجهات

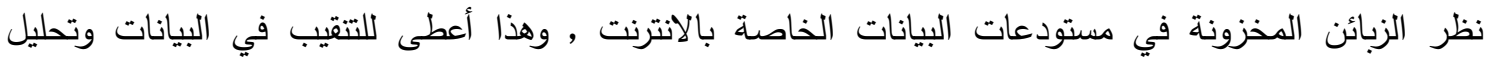

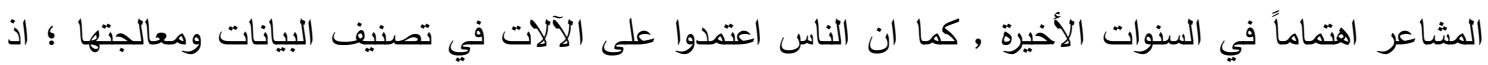

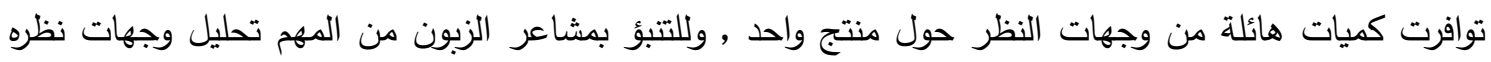

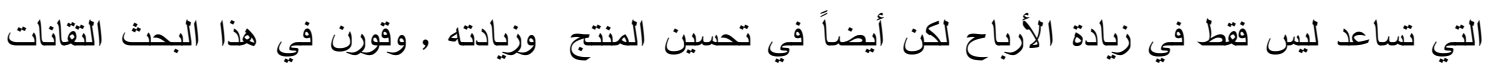

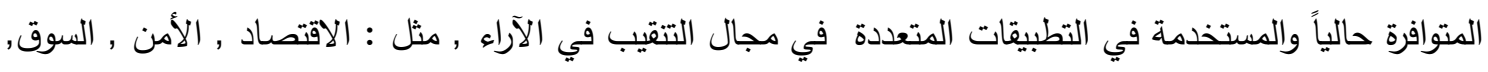
اكتثاف المحتوى غير المرغوب فيه في صفحات الانترنت , اتخاذ القرار , وتوقع نتائج الانتخابات .

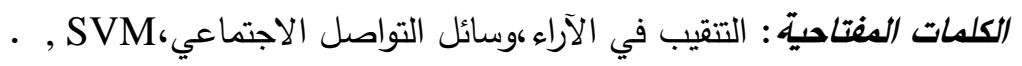
1 1

البشر مخلوقات متحيزة والآراء تهمهم ؛ لذلك فإن تحليل المشاعر (العواطف) يهدف إلى بناء نظام يقوم

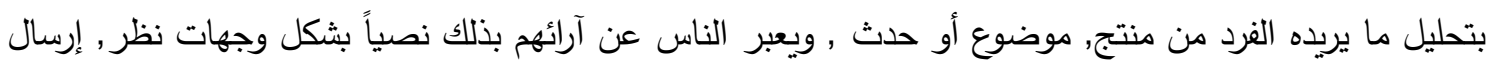
رسالة شخصية , تعليق , أو تغريدة. والتتيب في هذه الآراء المتوافرة لاى المستخدم عملية صعبة لكن الكن عملياً مفيدة

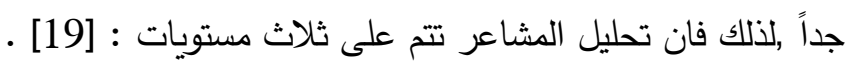


1- مستوى الوثيقة document level :يعمل على اختزال كامل المستتد إلى رأي واحد ,ولكن في أغلب الحالات

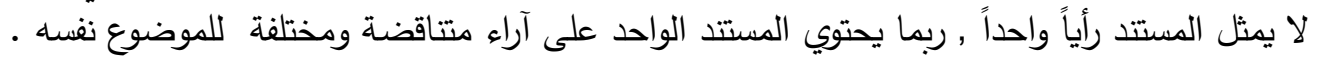

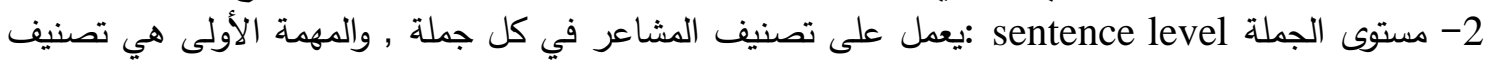
الجملة فيما إذا كانت تحتوي على الكيان تحت التمحيص( objective ) أو تحتوي كلمات صريحة تمثل هئل الرأي عن ذلك الكيان( subjective).

3- مستوى الخاصية attribute level :يهدف إلى تصنيف الخواص المختلفة للموضوع نفسه , والمهمة الأولى الذي

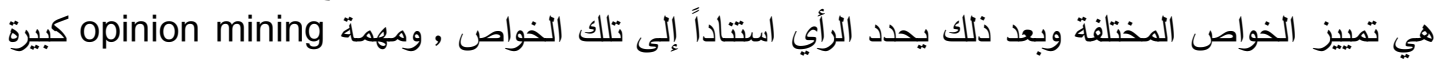

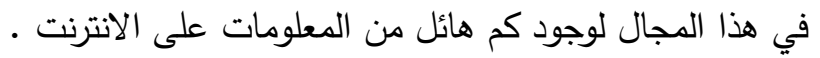

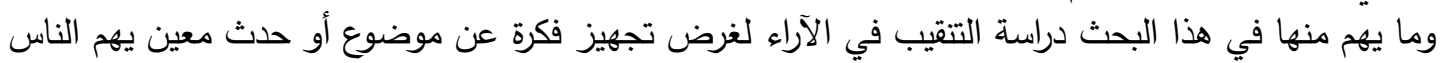

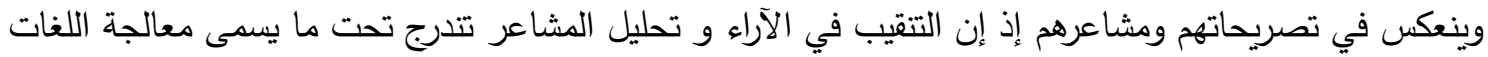

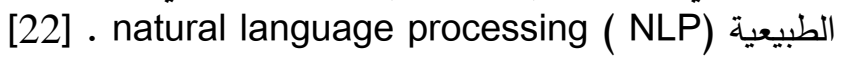
وللحصول على الآراء فعلينا بالإعلام الاجتماعي Social media ؛ لأنه واحد من المساحات المفتوحة للتعبير

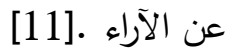
التتقيب في الآراء وتحليل المشاعر : إما يكون بتقانات معالجة اللغات الطبيعية NLP , أو باستخدام إحصائيات أو

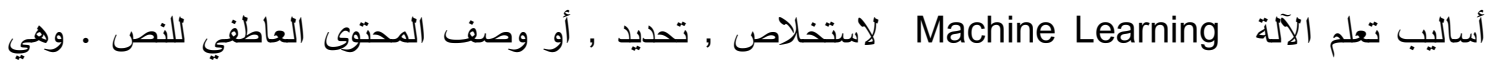
دراسة حسابية عن سياسة الناس , اتجاهاتهم , وجهات نظرهم وعواطفهم تجاه مواضيع معينة , منتجات, أفراد,

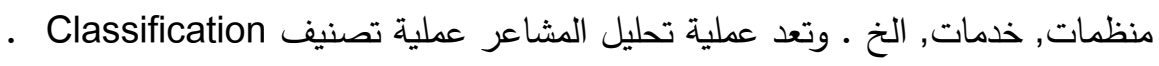
التتقيب في الآراء هو أحد فروع التتقيب في محتوى الانترنت Web Content Mining , والتتقيب في محتوى

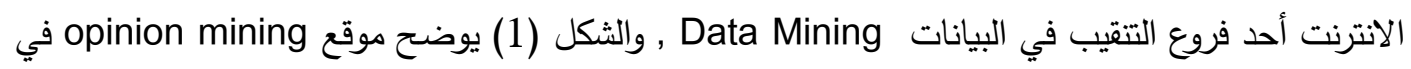

هيكلية Data Mining

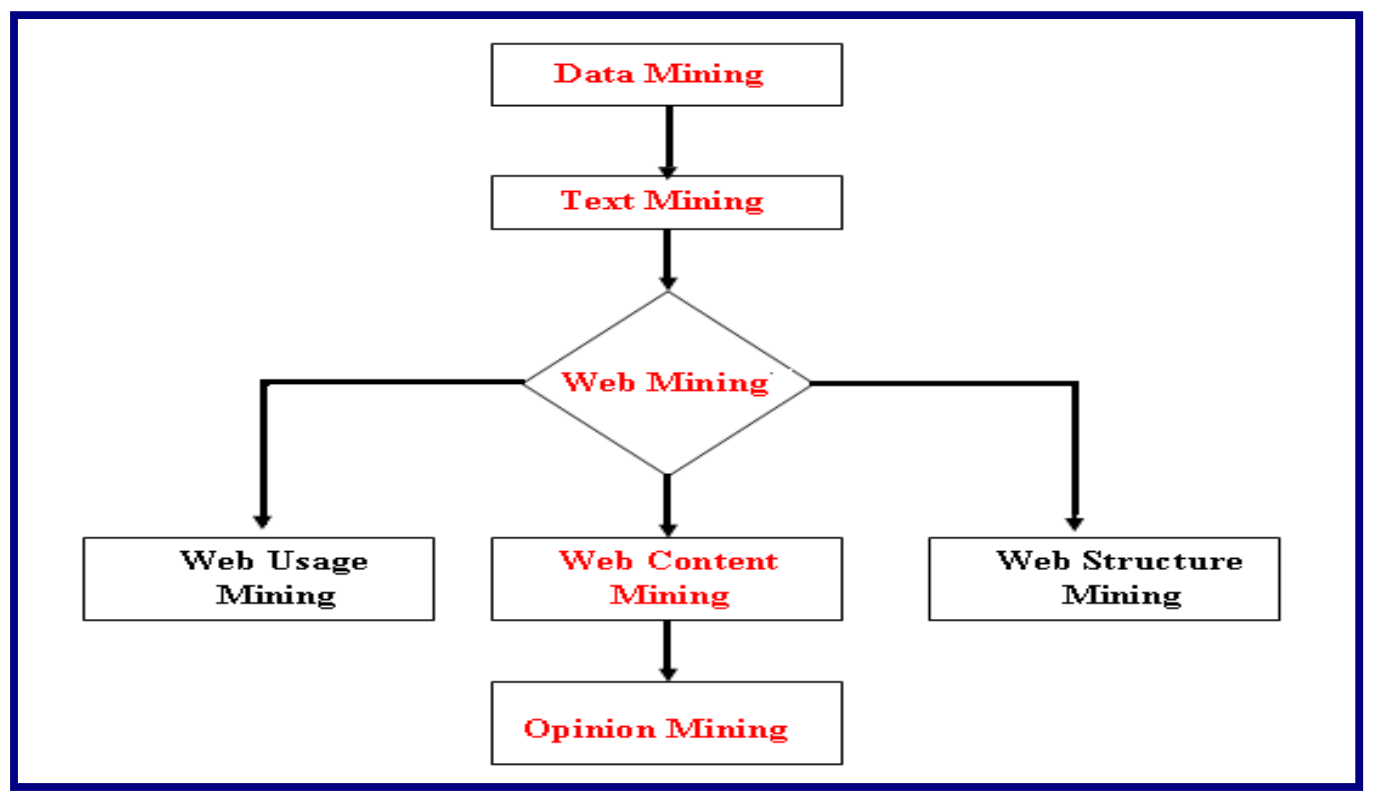

[20] Data Mining الشكل (1) هيكلية

تبين من البحث في opinion mining بأن هناك تحليلات أخرى تتعلق بالموضوع نفسه وقد ترد مستقلة

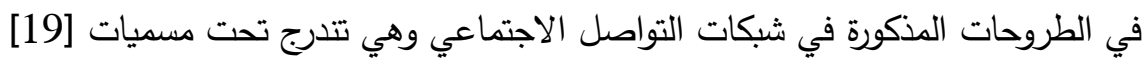
تحليل الموضوع Subjectivity Analysis . 
التتقيب في وجهات النظر Review Mining .

استخلاص المديح Appraisal Extraction .

ومكونات التتقيب في الآراء موضحة بالثكل (2) وتتضمن :

1. Object الموضوع موناء

2. مامل الرأي Opinion Holder.

3. Opinion الرأي

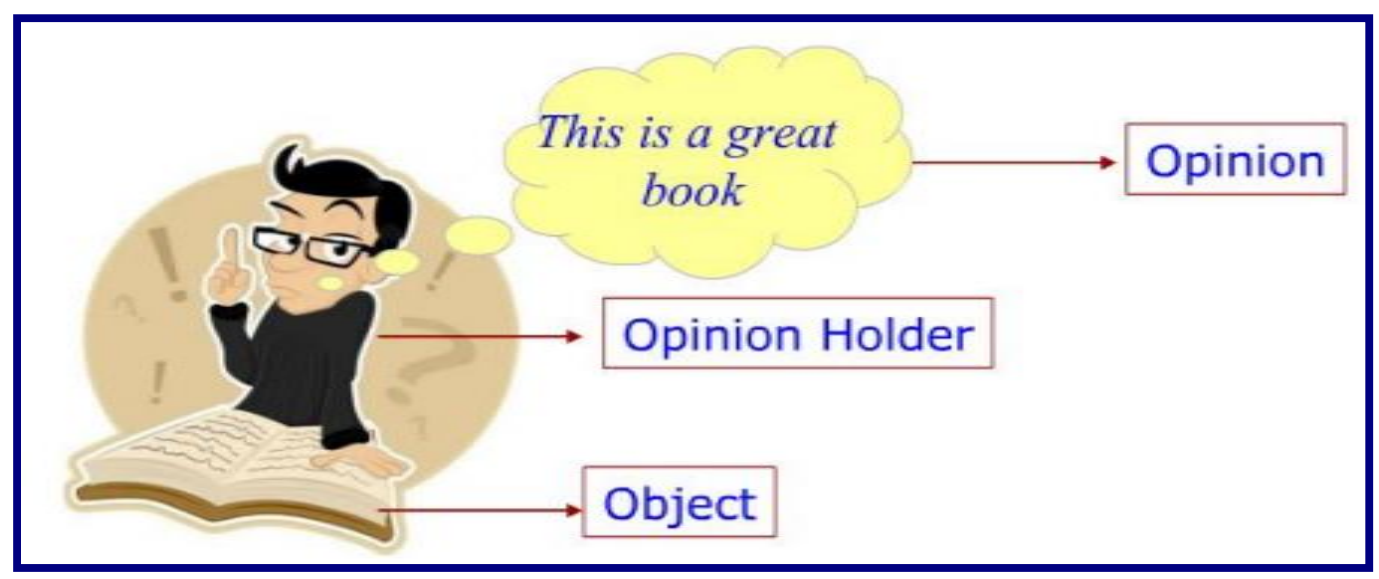

الثكل (2) : مكونات التتقيب في الآراء [11]

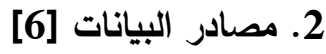

لكون الآراء مقدمة من الأشخاص , فان الثركات المنتجة تقوم بتقييم تلك الآراء بدراسة البيانات المسجلة في المقالات الثخصية , والمواقع التي فيها وجهات نظر , إضافة إلى محادثات الانترنت و المقالات الإخبارية .

\section{2 وجهات النظر Review}

يوجد الكثير من وجهات النظر التي يسجها المستخدم والموجودة على الانترنت بحيث تساعد الزبون على مئ

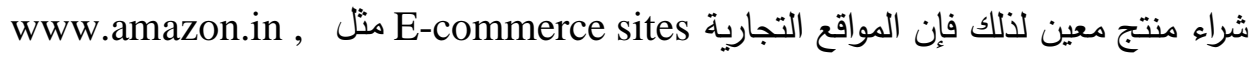
www.reviewcentre و www.flipkart.com

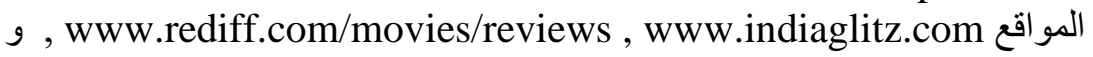

Www.yelp.com,www.burrp.com تمتلك آراء www.rottentomatoes.com عن المطاعم.

\section{2 محادثات الانترنت Web Discourse}

المقالات الثخصية (blog) أو صفحات الانترنت التي يسجل الأفراد آراءهم فيها ؛ إذ يربطون مواقعهم مع

آخرين , وتمتاز بالأسلوب البسيط والكلام الجاد في كتابة الرسائل وترسل للانترنت مما جعل عالم المعالات لاتلات

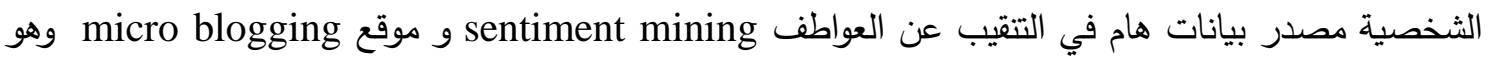
يفيض بالآراء الحاسمة في مصير نتائج الانتخابات , وهذه الآراء تستعمل في تصنيف العواطف ؛ إذ إن 
الناس توثق الأحداث اليومية في حياتها وتعبر عنها بالآراء , المشاعر و العواطف في مقالات شخصية على تويتر

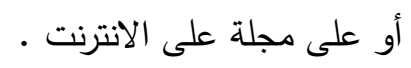

News Articles 3.2

إن مواقع الانترنت مثل www.thesun.co.uk, www.cnn.com و فيها مقالات إخبارية تسمح للمستخدمين والقُرًاء بالتعليق • وهذا يساعد في تسجيل آراء الناس في القضايا ذات الصلة والأهمية . معالات

3. تطبيقات التنقيب في الآراء وتحليل المشاعر هناك عدة تطبيقات تحتاج التنقيب في الآراء وتحليل المشاعر ومنها [16] Purchasing Product or Service شراء منتج أو شراء خدمة 1.3

عملية التتقيب في الآراء تساعد الأفراد في شراء منتج أو خدمة لشركة ما من دون الاعتماد على رأي المستشارين الخارجيين الذين يطلبون مبالغ مالية قد تكون كبيرة مقابل كل استثارة , وهذه نقطة توفير مالية مهمة

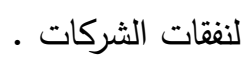

Quality Improvement in Product or service 2.3

تقوم الشركات و المصانع وحتى المنظمات والحكومات بالاستتاد إلى آراء الناس في تحسين خدماتها أو منتجاتها المقدمة لهم بما يتتاسب مع متطلباتهم واحتياجاتهم وتحديد مواطن الضعف والقوة في المنتج أو الخدمة ,

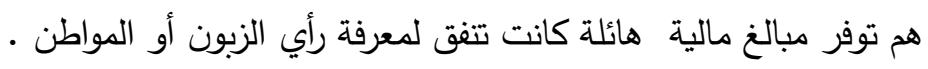

Marketing research 3.3

هي عملية تحديد ميول الزبون نحو منتجات أو خدمات بعينها ـ كذلك تحديد نجاح حملة الإعلانات بإطلاق منتج جديد , ودراسة ما يحتاجه الفرد من منتجات أو خدمات غير متوافرة في السوق , وإذا ما استغلت هذه الآراء اقتصادياً فإنها ذات منفعة كبيرة .

Detection of "flame" اكتثاف المشاعر الملتهبة 4.3

هذه النقطة مفيدة في تحديد الآراء والأفكار الخبيثة التي تثير الجدل والكراهية والانزعاج بين أفراد البلد

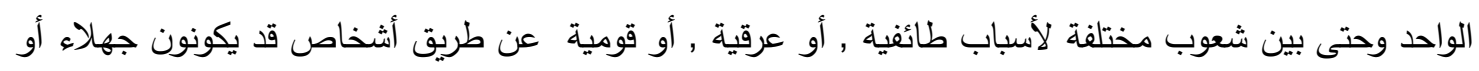

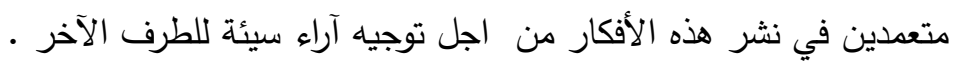

Opinion spam detection 5.3

ينثر الفرد ما يثاء من آراء وكتابات على الانترنت وقد يكون محتواها غير مهم • وقد قسمت عملية

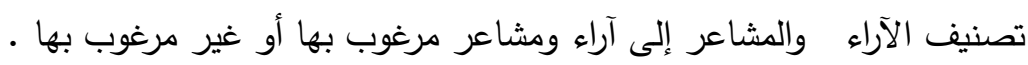


Decision Making 6.3

ساعدت عملية التتقيب الثركات والمنظمات والحكومات على اتخاذ قرارات مهمة وحاسمة أحياناً بإشراك

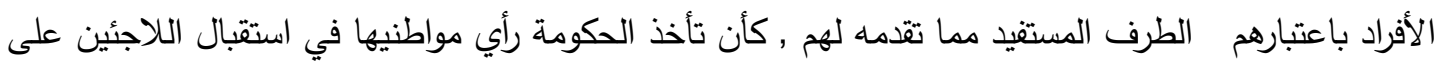
أراضيها وعلى ضوء النتائج تأخذ الحكومة القرار , أي ستسهم في صنع السياسة الخارجية تاخية .

Elections Expectation توقع نتائج الانتخابات 7.3

يساعد التتقيب في الآراء في توقع الفائز في الانتخابات العامة في البلاد بمقارنة آراء الناس عن أكثر

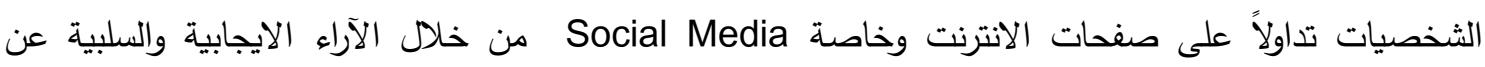

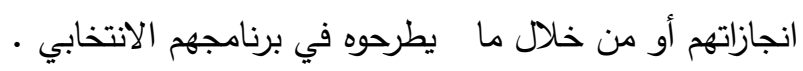

\section{Classification Methods [11] طرائق التصنيف}

يتضح من الجدول (1) بأن هناك ثلاثة طرائق استخدمت في تحليل المشاعر وهي :

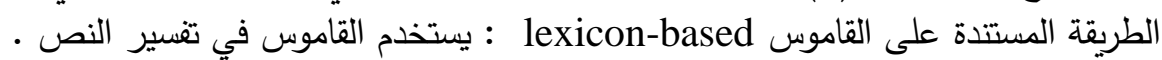

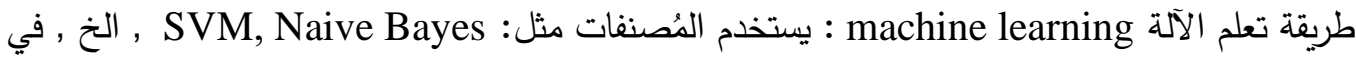

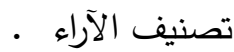

طريقة الأسلوب الهجين Hybrid Approach (الطريقة المستتدة على القاموس + طريقة تعلم الآلة ) . ومن الخوارزميات التي تكرر استخدامها في هذا المجال : [11]

SVM-Support Vector Machines 1.4

خوارزمية تستخدم للتصنيف ,الانحدار و تمييز الانماط. والهدف منها هو إيجاد أفضل دالة تصنيف وأيضاً

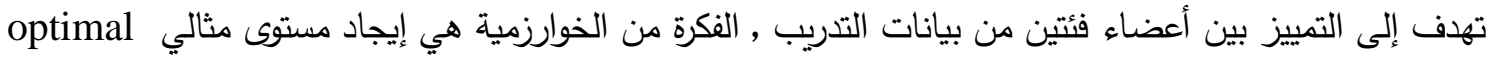

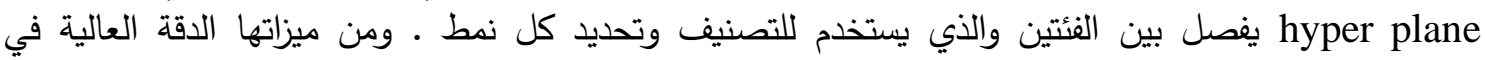
Image التصنيف وتطبق في مجالات واسعة منها تحديد فئات النص Text categorization تصنيف الصين الصورة classification Naive Bayes 2.4

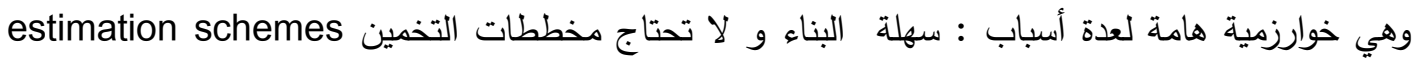
لأي متغيرات تكرارية معقدة , وقد تطبق بسهولة على مجموعة بيانات ضخمة واجة والهدف من الخوارزمية هو بناء

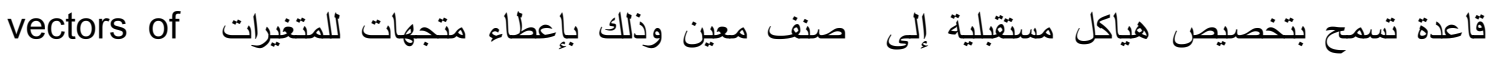

variable

K-Nearest Neighbor 3.4

تستخدم في التصنيف والانحدار وهي طريقة غير قياسية , إذ عندما تكون العينة المقدمة إلى الخوارزمية

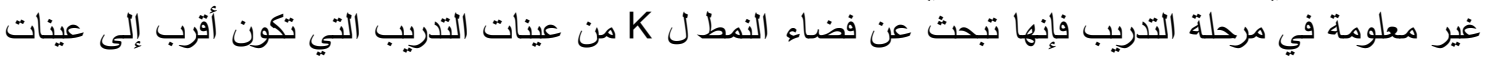
غير معلومة , وتعتمد كفاءة الخوارزمية على نحو رئيس على القيم التي تستخدم في الخوارزمية , وتمتاز هذها

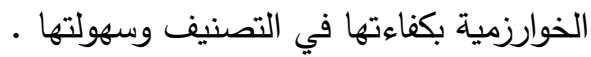

5VALUATION AND DESCRIPTION التقييم والوصف يقيم أداء الخوارزميات على أساس الصحة accuracy , والدقة precision , ومقياس F1-measure والاستدعاء recall , في هذا البحث تم التركيز على الصحة accuracy التي تم الحصول عليها من الخوارزميات

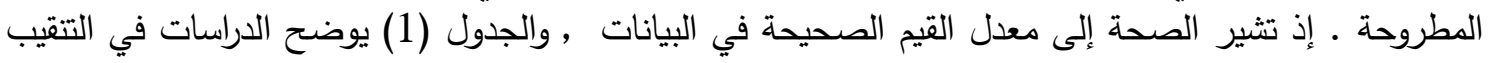
في الآراء وتحليل المشاعر (العواطف) باستخدام تقانات مختلفة. 
الجدول يحدد أيضاً مصادر البيانات المستخدمة , وبخصوص كفاءة الخوارزميات فمن الصعب توقع الأفضل

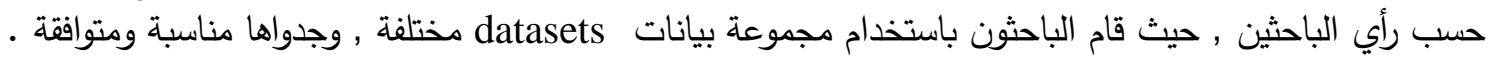
ومما تجدر الإثارة إليه بان بيانات البحوث الواردة في الجدول(1) جميعها باللغات غير العربية .

6. الخاتمة

إن التتقيب في الآراء والتحليل العاطفي هو حقل من التتقيب في البيانات Data Mining يستعمل في

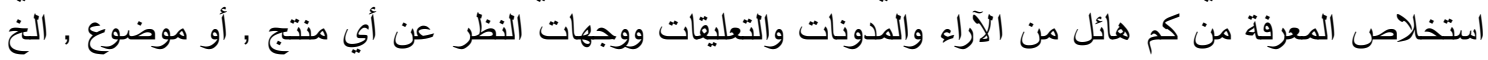

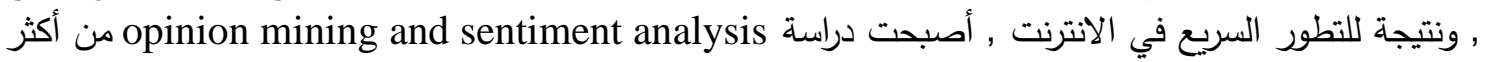

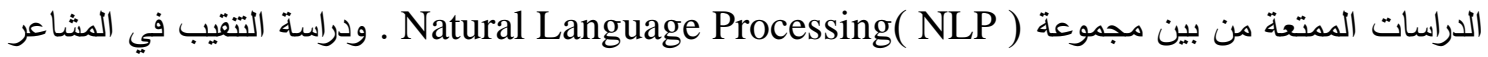

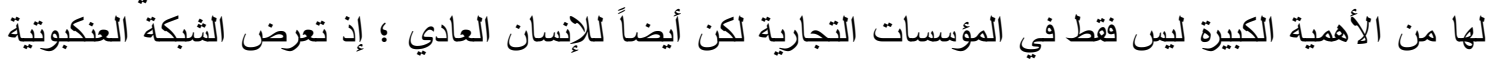

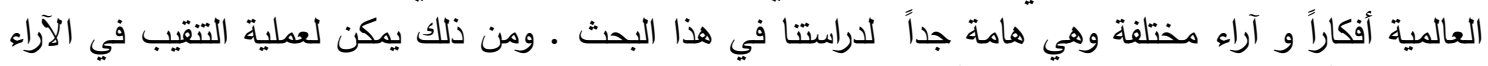

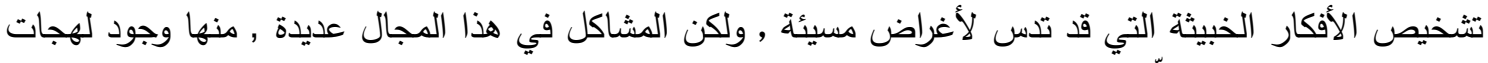
محلية ومناطقية مختلفة للّغة الواحدة , ولكل منها معانٍ وقواعد مختلفة تستخدم في صياغة تلكي لكن الجمل.

\begin{tabular}{|c|c|c|c|c|c|}
\hline الصحة & مصدر البيانات & التقانة الستخذمة & السنة & اسم المؤلف & \\
\hline \% 90 SVM & $\begin{array}{l}\text { ChnSentiC } \\
\text { مشاعر صـــrp }\end{array}$ & $\begin{array}{c}\text { مُصَّن , SVM } \\
\text { K-, Centroid } \\
\text { Nearest } \\
\text { neighbour } \\
\text { Winnow }\end{array}$ & 2008 & Tan & [25] \\
\hline$\% 89$ & 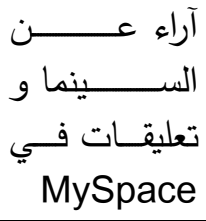 & Hybrid, SVM & 2009 & Prabowo & [14] \\
\hline$\% 91.21$ & Blogs & تصنيف Bayesian & 2009 & Melville & [12] \\
\hline$\% 91.51$ & و آراء عن منتج & SVM & 2011 & $\begin{array}{l}\text { Rushdi } \\
\text { Saleh M. }\end{array}$ & [19] \\
\hline$\% 86.6$ & 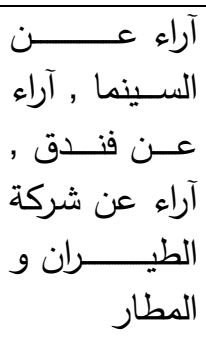 & Naïve Bayes & 2011 & Khan & [10] \\
\hline$\% 61$ & Amazon آراء من موقع & $\begin{array}{c}\text { Naïve } \\
\text { bayes ,Maximum } \\
\text { entropy ,SVM }\end{array}$ & 2011 & Xia & [27] \\
\hline$\% 93$ & آراء كانتوني & Naïve Bayes & 2011 & Zhang & [28] \\
\hline
\end{tabular}




\begin{tabular}{|c|c|c|c|c|c|c|c|}
\hline \multicolumn{2}{|c|}{ الصحة 79.66} & التقنية & \multirow{5}{*}{ الراءتف عـــــــن } & \multirow{5}{*}{$\begin{array}{c}\text {, Naive Bayes } \\
\text { Modified , KNN } \\
\text { g-Means +NB } \\
\text { Modified K- } \\
+ \text { Means + NB } \\
\text { KNN }\end{array}$} & \multirow[t]{5}{*}{2011} & \multirow[t]{5}{*}{ B. Chen } & \multirow[t]{5}{*}{ [4] } \\
\hline \multicolumn{2}{|c|}{ \% 79.66} & $\begin{array}{l}\text { Naive } \\
\text { Bayes }\end{array}$ & & & & & \\
\hline \multicolumn{2}{|c|}{$\% 83.59$} & KNN & & & & & \\
\hline \multicolumn{2}{|c|}{$\% 89$} & $\begin{array}{r}\text { Modified } \\
\text { K-Means } \\
+\mathrm{NB}\end{array}$ & & & & & \\
\hline \multicolumn{2}{|c|}{$\% 91$} & $\begin{array}{l}\text { Modified } \\
\text { K-Means } \\
+\quad+\text { NB } \\
\text { KNN }\end{array}$ & & & & & \\
\hline \multicolumn{3}{|c|}{$\% 77$ SVM-PSO } & تويتر & $\begin{array}{c}\text { SVM- g SVM } \\
\text { PSO }\end{array}$ & 2012 & $\begin{array}{l}\text {, Basari } \\
\text { gussin } \\
\text { Ananta }\end{array}$ & [2] \\
\hline \multicolumn{3}{|r|}{$\% 74.8268$} & الفيسبوك & SVM & 2012 & $\begin{array}{l}\text { A. } \\
\text { Shrivatava } \\
\text { B. Pant g }\end{array}$ & [23] \\
\hline \multicolumn{2}{|c|}{ with } & $\begin{array}{r}\text { Polykernel } \\
\% 87.00\end{array}$ & \multirow[t]{3}{*}{ 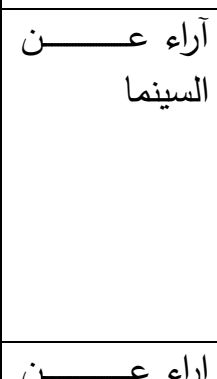 } & \begin{tabular}{|rr} 
SVM & with \\
& Polykernel
\end{tabular} & \multirow[t]{3}{*}{2014} & \multirow{3}{*}{$\begin{array}{l}\text { K.SARAS } \\
\text { WATHI } \\
\text { Dr. } \\
\text { A.TAMILA } \\
\text { RASI }\end{array}$} & \multirow[t]{3}{*}{ [7] } \\
\hline with & \multicolumn{2}{|c|}{$\begin{array}{r}\text { RBF Kernel } \\
\% 73.33\end{array}$} & & $\begin{array}{r}\text { SVM with RBF } \\
\text { Kernel }\end{array}$ & & & \\
\hline Bagging & \multicolumn{2}{|c|}{$\begin{array}{r}\text { with SVM } \\
\% 88.00 \\
\end{array}$} & & $\begin{array}{lr}\text { Bagging } & \text { with } \\
& \text { SVM }\end{array}$ & & & \\
\hline الصيل الصـــة & | & الأسلوب & الكاميراء عـــــن & $\begin{array}{c}\text { Domain ,SVM } \\
\text { Lexicons+MPQA+ } \\
\text { SVM }\end{array}$ & 2014 & $\begin{array}{l}\text {, Rastogi } \\
\text { Singhal } \\
\text { Kumar }\end{array}$ & {$[18]$} \\
\hline \multicolumn{3}{|c|}{$\% 63$} & 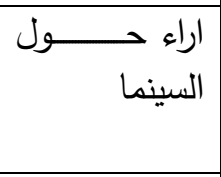 & \begin{tabular}{rr}
\multicolumn{2}{c}{ Document based } \\
opinion mining \\
system \\
\end{tabular} & 2014 & $\begin{array}{l}\text { Sharma } \\
\text { و Nigam } \\
\text { Jain } \\
\end{array}$ & [22] \\
\hline \multicolumn{2}{|c|}{ الصحة } & التقنية & \multirow{4}{*}{ الهاتف النقال } & \multirow{4}{*}{$\begin{array}{lr}\text { Naive } & \text { SVM } \\
9 & \text { Bayes } \\
\text { NB+Modified } & \text { K- } \\
& \text { Means }\end{array}$} & \multirow[t]{4}{*}{2015} & \multirow{4}{*}{$\begin{array}{l}\text { S. gPreety } \\
\text { Dahiya }\end{array}$} & \multirow[t]{4}{*}[15]{} \\
\hline$\% 79$. & & $\begin{array}{l}\text { Naive } \\
\text { Bayes }\end{array}$ & & & & & \\
\hline$\% 83$. & & SVM & & & & & \\
\hline$\%$ & 89 & $\begin{array}{l}\text { NB+Modifi } \\
\text { ed } \begin{array}{r}\mathrm{K}- \\
\text { Means }\end{array}\end{array}$ & & & & & \\
\hline حة & الص - الص & التقنية & تغريدات & , Naïve Bayes & 2015 & & [9] \\
\hline$\% 81$. & & $\begin{array}{l}\text { Naïve } \\
\text { Bayes }\end{array}$ & & $\begin{array}{l}\text { Multinomial Nave } \\
\text { Decision , Bayes }\end{array}$ & & Kethavath & \\
\hline$\% 89$. & & $\begin{array}{r}\text { Multinomi } \\
\text { al } \begin{array}{r}\text { Nave } \\
\text { Bayes }\end{array}\end{array}$ & & SVM g Tree & & & \\
\hline
\end{tabular}




\begin{tabular}{|c|c|c|c|c|c|c|}
\hline$\% 80.08$ & $\begin{array}{r}\text { Decision } \\
\text { Tree }\end{array}$ & & & & & \\
\hline$\% 94.45$ & SVM & & & & & \\
\hline الصحة & التقنية & \multirow{5}{*}{ تويتر } & \multirow{5}{*}{$\begin{array}{l}\text {, Naïve Bayes } \\
\text { Max , SVM } \\
\text { J48 Entropy }\end{array}$} & \multirow[t]{5}{*}{2015} & \multirow{5}{*}{$\begin{array}{l}\text { Dr. Raj.S } \\
\text { Suresh. }\end{array}$} & \multirow[t]{5}{*}{ [24] } \\
\hline$\% 76$ & $\begin{array}{l}\text { Naïve } \\
\text { Bayes }\end{array}$ & & & & & \\
\hline$\% 88$ & SVM & & & & & \\
\hline$\% 89.2$ & $\begin{array}{r}\text { Max } \\
\text { Entropy }\end{array}$ & & & & & \\
\hline$\% 92$ & J48 & & & & & \\
\hline & $\% 97.54$ & توبيانـــات مـــن & SVM & 2016 & $\begin{array}{l}\text { Bholane D } \\
\text { Gore },\end{array}$ & [3] \\
\hline & $\% 93.7$ & $\begin{array}{r}\text { Weka } \\
\text { software } \\
\text { عن السينمة بيانـات }\end{array}$ & SVM & 2016 & $\begin{array}{l}\text {, da Rocha } \\
\text { وacheco } \\
\text { Mendonza }\end{array}$ & [17] \\
\hline & $\% 84$ & يوتيوب & SVM & 2017 & $\begin{array}{l}\text { Tanesab } \\
\text { Sembiring } \\
\text { Purnomo }\end{array}$ & [26] \\
\hline الصحة & التقنية & \multirow{5}{*}{ الهاءت عــــــن النقال } & \multirow{5}{*}{$\begin{array}{lr}\text { Naive } & \text { Bayes } \\
\text { Modified } & \text { KNN } \\
\text { g K-Means } & + \text { NB } \\
\text { Modified } & \text { K- } \\
+ \text { Means } & + \text { NB } \\
\text { KNN } & \end{array}$} & \multirow[t]{5}{*}{2017} & \multirow{5}{*}{$\begin{array}{l}\text { Aggarwal } \\
\text { L. Gupta و }\end{array}$} & \multirow[t]{5}{*}[1]{} \\
\hline 79.66 & $\begin{array}{l}\text { Naive } \\
\text { Bayes }\end{array}$ & & & & & \\
\hline 83.59 & KNN & & & & & \\
\hline 89 & $\begin{array}{r}\text { Modified } \\
\text { K-Means } \\
+\mathrm{NB}\end{array}$ & & & & & \\
\hline 91 & $\begin{array}{r}\text { Modified } \\
\text { K-Means } \\
+\quad+\text { NB } \\
\text { KNN }\end{array}$ & & & & & \\
\hline
\end{tabular}




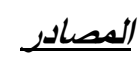

[1] Aggarwal R. And Gupta L. , A Hybrid Approach For Sentiment Analysis Using Classification Algorithm , International Journal Of Computer Science And Mobile Computing , Page : 149-157,2017.

[2] Basari A.S.H., Hussin B., And Ananta I.G.P. , Opinion Mining Of Movie Review Using Hybrid Method Of Support Vector Machine And Particle Swarm Optimization, Malaysian Technical Universities Conference On Engineering And Technology (Mucet), Page: 545-552,2012.

[3] Bholane Savita D. And Prof. Deipali Gore, Sentiment Analysis On Twitter Data Using Support Vector Machine , International Journal Of Computer Science Trends And Technology (I Jcst), Page: 365-370, 2016.

[4] Chen B. , Topic Oriented Evolution And Sentiment Analysis , The Pennsylvania State University, Information Sciences And Technology, Doctor Of Philosophy , Page : 1-126,2011 .

[5] Gaur M. And Pruthi J. , A Survey On Sentiment Analysis And Opinion Mining, International Journal Of Current Engineering And Technology, Page : 445,2017 .

[6] Govindarajan M., Romina M., A Survey Of Classification Methods And Applications For Sentiment Analysis , The International Journal Of Engineering And Science (Ijes), Page: 12, 2013.

[7] K.Saraswathi And Dr. A.Tamilarasi , Investigation Of Support Vector Machine Classifier For Opinion Mining , Journal Of Theoretical And Applied Information Technology, Page : 291-296, 2014.

[8] Kang H., Yoo S.J. , Han D., Senti-Lexicon And Improved Naïve Bayes Algorithms For Sentiment Analysis Of Restaurant Reviews. Expert Systems With Applications , Page : $39,2012$.

[9] Kethavath S. , Classi_Cation Of Sentiment Analysis On Tweets Using Machine Learning Techniques , Page : 1-28, 2015.

[10] Khan A., Baharudin B. And Khan K. , Sentiment Classification Using Sentence-Level Lexical Based Semantic Orientation Of Online Reviews , Trends In Applied Sciences Research, Page: 1141-1157 ,2011.

[11] Kulkarni A.A., Hundekar V.A., Sannakki S.S. And Rajpurohit V.S., Survey On Opinion Mining Algorithms And Applications, International Journal Of Computer Techniques, Page :9, 2017.

[12] Melville And Gryc W., Sentiment Analysis Of Blogs By Combining Lexical Knowledge With Text Classification, Kdd ${ }^{e e} 09$, June 28-July 1, 2009, Paris, 
France.Copyright 2009 Acm 978-1-60558-495-9/09/06 , 2009.

[13] Ms. Sahare S.A., A Survey Paper On Opinion Mining And Sentiment Analysis, Ijariie, Page : $5075,2017$.

[14] Prabowo R., Thelwall M., Sentiment Analysis: A Combined Approach . , Journal Of Informetrics ,Page : 143-157,2009 .

[15] Preety And Dahiya S. , Sentiment Analysis Using Svm And Naïve Bayes Algorithm , International Journal Of Computer Science And Mobile Computing, Page : 212-219, 2015 .

[16] Rababah O.M., Hwaitat A.K. And Al Qudah D.A., Sentiment Analysis As A Way Of Web Optimization, Scientific Research And Essays , Page : 90-91, 2016.

[17] Rocha R.S.C.D. , Pacheco M.A. And Mendonza L.A.F., Sentiment Analisis On Web-Based Reviews Using Data Mining And Support Vector Machine , Int'l Conf. Information And Knowledge Engineering, Page : 113-117, 2016.

[18] S.S.K. Rastogi , Singhal R. And Kumar A. , An Improved Sentiment Classification Using Lexicon Into Svm , International Journal Of Computer Applications , Page : 37-42, 2014.

[19] Saleh M.R., Martín-Valdivia M.T., Montejo-Ráez A. And Ureña-López L.A. , Experiments With Svm To Classify Opinions In Different Domains , Expert Systems With Applications, Page : 14799-14804, 2011.

[20] Seerat B. And Azam F., Opinion Mining: Issues And Challenges (A Survey), International Journal Of Computer Applications, Page : 42 , 2012.

[21] Sharma R., Nigam S. And Jain R. Opinion Mining Of Movie Reviews At Document Level, International Journal On Information Theory (Ijit), Page : $13-21,2014$.

[22] Sharma S.P., Dr. Tiwari R. And Dr. Prasad R., Opinion Mining And Sentiment Analysis On Customer Review Documents- A Survey , International Journal Of Advanced Research In Computer And Communication Engineering, Page : 156, 2017.

[23] Shrivatava A. And Pant B. , Opinion Extraction And Classification Of Real Time Facebook Status, Global Journal Of Computer Science And Technology, Page : 35-39,2012 .

[24] Suresh H. And Dr. Raj.S , Analysis Of Machine Learning Techniques For Opinion Mining, International Journal Of Advanced Research, Page : 375 $381,2015$.

[25] Tan S., Zhang J., An Empirical Study Of Sentiment Analysis For Chinese 
Documents , Expert Systems With Applications ,Page : 2622-2629, 2008.

[26] Tanesab F.I., Sembiring I. And Purnomo H.D. , Sentiment Analysis Model Based On Youtube Comment Using Support Vector Machine, International Journal Of Computer Science And Software Engineering (Ijcsse), Page: 180185,2017 .

[27] Xia R., Zong C. ,And Li S. , Ensemble Of Feature Sets And Classification Algorithms For Sentiment Classification, Information Sciences, Page : $1138-1152,2011$.

[28] Zhang Z., Ye Q., Zhang Z., And Li Y., Sentiment Classification Of Internet Restaurant Reviews Written In Cantonese, Expert Systems With Applications , Page : 7674-7682, 2011. 